\title{
Pseudospectral contracted configuration interaction from a generalized valence bond reference
}

\author{
Robert B. Murphy and Richard A. Friesner \\ Department of Chemistry, Columbia University, New York, New York 10027 \\ Murco N. Ringnalda \\ Schrödinger Inc., 80 South Lake Avenue, Suite 735 Pasadena, California 91101 \\ William A. Goddard III \\ Materials and Molecular Simulation Center, Beckman Institute (134-74), Division of Chemistry and \\ Chemical Engineering, California Institute of Technology, Pasadena, California 91125
}

(Received 28 March 1994; accepted 29 April 1994)

\begin{abstract}
A multireference configuration interaction method is presented based upon pseudospectral integration and a novel generalized valence bond referenced contraction procedure. The combination of these approaches is shown to allow for unprecedented multiconfiguration self-consistent-field calculations on large molecules.
\end{abstract}

\section{INTRODUCTION}

Substantial progress has been made in the development of accurate self-consistent multiconfigurational (MCSCF) wave functions. ${ }^{1}$ In particular, it has been shown that multireference $\mathrm{CI}(\mathrm{MRCI})^{2}$ and more recently multireference perturbation theory (MRPT) ${ }^{3-6}$ using these MCSCF references leads to quite accurate predictions for a wide variety of molecular properties. However, the computational cost of these MCSCF based methods grows very quickly with the size of the molecule thus limiting studies at the MRCI or multiconfigurational perturbation theory level to the order of 10 to 20 electrons. The two computational bottlenecks of conventional methods which have prevented the applications of highly correlated methods to large molecules are the unfavorable scaling with basis size of the two-electron integral evalution over orbitals and the rapid growth in the length of the multiconfigurational expansion with the number of active orbitals.

In this paper we present new methods for significantly reducing the cost of two-electron integral evaluation via pseudospectral integration techniques and a novel contraction procedure for reducing the length of $\mathrm{CI}$ expansions generated from a generalized valence bond (GVB) reference. These two techniques introduce the possibility of correlated calculations of unprecedented size.

The pseudopsectral integration method, developed by Friesner and co-workers, ${ }^{7-12}$ uses a Gaussian basis set and a numerical grid together to obtain two-electron integrals each involving four functions, two active orbitals, and two basis functions. For $N_{\text {bas }}$ basis functions this procedure scales as $N_{\text {bas }}^{3}$ as compared to the conventional $N_{\text {bas }}^{4}$ scaling of evaluating integrals over basis functions. Furthermore, a fast twoindex transform of the pseudopsectral integrals gives the necessary integrals over orbitals for a CI calculation. In contrast, the conventional methodology requires an expensive ( $N_{\text {bas }}^{5}$ process) four-index transform from the basis set to the orbital space. The pseudospectral integration method, while an approximation to the conventional analytic integration, has been shown ${ }^{11}$ to give total electronic energies to within $0.1 \mathrm{kcal} / \mathrm{mol}$ of conventional methods for Hartree-Fock and
GVB wave functions. We show here that the same accuracy is obtainable for a MRCI wave function constructed from a GVB reference.

The correlated wave function we develop here is constructed from intrapair excitations of a GVB perfect pairing (GVB-PP) reference wave function where perfect pairing refers to the use of a single valence bond spin coupling among pairs of bonded orbitals. This so-called "restricted CI" (RCI) wave function uses an active-orbital space of the two natural orbitals of each GVB-PP pair. The RCI wave function in what will be referred to as its "uncontracted" form was first considered by Harding and Goddard. ${ }^{13}$ The RCI wave function, though less complete than a complete-active-space (CASSCF) $)^{14,15}$ expansion defined in the same active orbital space, has been shown ${ }^{4,5,16}$ to give energies and properties when used as a reference for subsequent MRCI or MRPT which are nearly the same as those obtained with a CASSCF reference.

Although the uncontracted RCI wave function contains fewer configurations and is hence more manageable than a CASSCF expansion, the length of this expansion grows beyond a million configurations for 20 electrons. The key to making the RCI expansion tractable for large systems is shown here to lie in the structure of the multiconfigurational GVB-PP reference wave function. The spatial part of the GVB-PP wave function is defined as a single product of pairwise overlapping local orbitals. Each pair of bonded local orbitals is expressed as a linear combination of mutually orthogonal local natural orbitals with the two coefficients of this nonorthogonal to orthogonal transformation being variational parameters. In the natural orbital basis the wave function describing $N$ GVB pairs is an expansion in $2^{N}$ closedshell deteminants. However, the $\mathrm{CI}$ coefficients of this expansion are functionals of the $2 N$ orbital coefficients defining the transformation from the local overlapping GVB orbitals to the orthogonal natural orbitals. Therefore, the GVB-PP wave function represents a contracted CI expansion in which the contraction is defined by the shapes of the local orbitals. For a large number of pairs, the calculation of the $2 N$ orbital coefficients ${ }^{17}$ is far simpler than diagonalization 
of a large $2^{N}$ dimensional matrix. Most importantly, the GVB-PP energy is within $0.02 \mathrm{kcal} / \mathrm{mol}$ of the fully variational $2^{N}$ configuration $\mathrm{CI}$ for a series of small molecule test cases we have examined.

In this paper we extend this orbital based contraction to the RCI wave function, and in principle, to more general MRCI and MRPT expansions from a reference expressed in terms of GVB pairs. The RCI contraction procedure consists of two parts. First, the RCI expansion is defined by making excitations from the orbital contracted, multiconfigurational GVB-PP expansion rather than by making excitations of the individual $2^{N}$ configurations contained in the GVB expansion. This definition is analogous to the MRCI contraction procedure commonly used ${ }^{2}$ by other authors, with the new aspect that the reference itself is also contracted. Each variationally weighted RCI configuration is a sum of many uncontracted configurations, Second, as with GVB-PP, the RCI CI coefficients are functionals of the GVB-PP pair coefficients. As demonstrated below, this contraction procedure leads to very large reductions in the size of the CI matrix with little loss in accuracy relative to the corresponding uncontracted expansion. Furthermore, matrix elements among contracted RCI configurations are easily evaluated by replacing the common unexcited GVB-PP pairs of interacting configurations by a generalized Fock operator representing the mean field of the unexcited GVB-PP pairs. This correlated mean field replaces a large fraction of the two-electron matrix elements by a rapidly evaluated single-particle operator.

We develop in some detail the contracted pseudospectral RCI wave function outlined above. Examples of the high accuracy of the contraction approximation relative to the uncontracted $\mathrm{CI}$ are provided. Pseudospectral integration is shown to cause negligible errors relative to conventional analytic integration. Finally, we present test calculations on diatomic molecules and CPU timings for larger molecules.

\section{GVB REFERENCE WAVE FUNCTION}

We first discuss the GVB reference wave function to point out the computational efficiencies of this multiconfigurational expansion and to motivate the use of these efficiencies in constructing correlated wave functions from a GVB reference. A more extensive discussion of the optimization of GVB-PP wave functions can be found in Ref. 17. The reference GVB perfect-pairing (GVB-PP) wave function $\Psi^{\mathrm{GVB}-\mathrm{PP}}$ is composed of $N_{\text {gvb }}$ pairs of singlet-coupled local orbitals $\varphi_{i 1}, \varphi_{i 2}$ and a closed-shell core. The perfect-pairing nomenclature refers to the use of only the single valence bond spin coupling among the orbitals.

$$
\begin{aligned}
\Psi^{\mathrm{GVB}-\mathrm{PP}}= & \mathcal{\partial}\left[\{\operatorname{core}\}\left\{\varphi_{11} \varphi_{12} \ldots \varphi_{N_{1}} \varphi_{N_{2}}\right\}\right. \\
& \times\{(\alpha \beta-\beta \alpha) \ldots(\alpha \beta-\beta \alpha)\}],
\end{aligned}
$$

with $\&$ the antisymmetrizing operator. The local orbital pairs $\varphi_{i 1}, \varphi_{i 2}$ mutually overlap, however, a strongorthogonality restriction is applied which forces orbitals of different pairs to be mutually orthogonal. As shown by Goddard et al., ${ }^{17}$ this restriction and a computationally more use- ful form of $\Psi^{\mathrm{GVB}-\mathrm{PP}}$ can be represented by expanding each local orbital pair $\varphi_{i 1}, \varphi_{i 2}$ in terms of mutually orthogonal natural orbitals $\phi_{i 1}, \phi_{i 2}$ via the relations,

$$
\begin{aligned}
& \varphi_{i 1}=\left(\sigma_{i 1}^{1 / 2} \phi_{i 1}+\sigma_{i 2}^{1 / 2} \phi_{i 2}\right) /\left(\sigma_{i 1}+\sigma_{i 2}\right)^{1 / 2}, \\
& \varphi_{i 2}=\left(\sigma_{i 1}^{1 / 2} \phi_{i 1}-\sigma_{i 2}^{1 / 2} \phi_{i 2}\right) /\left(\sigma_{i 1}+\sigma_{i 2}\right)^{1 / 2}, \\
& \sigma_{n m}>0 ; \quad \sigma_{i 1}^{2}+\sigma_{i 2}^{2}=1,
\end{aligned}
$$

with the pairwise normalization conditions of the last equation. The natural orbital pairs $\phi_{i 1}, \phi_{i 2}$ are generally well localized between a pair of atoms with $\phi_{i 1}$ of the "bonding" type and $\phi_{i 2}$ having more nodal or "antibonding" character. This simple transformation puts $\Psi^{\mathrm{GVB}-\mathrm{PP}}$ into the form,

$$
\begin{aligned}
\Psi^{\mathrm{GVB}-\mathrm{PP}}= & \sigma\left[\{ \operatorname { c o r e } \} \left\{\left({ }^{*} \sigma_{11} \phi_{11}^{2}-\sigma_{12} \phi_{12}^{2}\right) \ldots\right.\right. \\
& \left.\left.\left(\sigma_{N_{1}} \phi_{N_{1}}^{2}-\sigma_{N_{2}} \phi_{N_{2}}^{2}\right)\right\}\{\alpha \beta \ldots \alpha \beta\}\right] .
\end{aligned}
$$

Expansion of this expression shows that $\Psi^{\mathrm{GVB}-\mathrm{PP}}$ describing $N_{\text {gvb }}$ pairs is an expansion in $2^{N_{\text {gvb }}}$ closed-shell configuration-state functions $\gamma_{i}$, with CI coefficients $D_{i}$ defined as functionals of the pair coefficients $\left\{\sigma_{n m}\right\}$

$$
\begin{aligned}
\Psi^{\mathrm{GVB}-\mathrm{PP}} & =\sum_{i=1}^{2^{N_{\mathrm{gvb}}}} D_{i}\left(\left\{\sigma_{n m}\right\}\right)\left[\left(\prod_{k}^{N_{\mathrm{gvb}}} \phi_{i k} \phi_{i k}\right)(\alpha \beta \ldots \alpha \beta)\right] \\
& =\sum_{i=1}^{2_{\mathrm{gvb}}} D_{i}\left(\left\{\sigma_{n m}\right\}\right) \gamma_{i}
\end{aligned}
$$

The orbital coefficients $\left\{\sigma_{n m}\right\}$ are iteratively solved for Ref. 17 by diagonalizing operators $Y^{i}$ for each pair $i$ in the basis of the two natural orbitals $\phi_{i 1}, \phi_{i 2}$ of pair $i$,

$$
\begin{aligned}
& Y^{i} \boldsymbol{\sigma}_{i}=\epsilon_{i} \boldsymbol{\sigma}_{i}, \\
& Y_{n n}^{i}=h_{n n}+\frac{1}{2}(n n \mid n n)+\sum_{k \neq i} \sigma_{k}^{2}(2(k k \mid n n)-(k n \mid n k)), \\
& Y_{n m}=\frac{1}{2}(n m \mid m n), \quad \text { for } n \neq m .
\end{aligned}
$$

The sum on $k$ is over orbitals in the other pairs. For the GVB-PP reference, the lowest energy root $\left(\sigma_{i 1},-\sigma_{i 2}\right)$ of the two possible roots is selected, with the complementary root of the two-by-two space simply being $\left(\sigma_{i 2}, \sigma_{i 1}\right)$.

The important point is that the $2^{N_{\text {gvb }}} \mathrm{CI}$ coefficients' $D_{i}$ of Eq. (4) are represented in terms of only $2 N_{\text {gvb }}$ coefficients $\left\{\sigma_{n m}\right\}$. One may refer to this reduction or contraction in the number of variational parameters as an orbital based contraction since the orbital expansion coefficients $\left\{\sigma_{n m}\right\}$ define the contraction. For large systems, the iterative determination of these $2 N_{\mathrm{gvb}}$ terms $\sigma_{n m}$ is computationally faster than a diagonalization in the $2^{N_{\mathrm{gvb}}}$ configuration space required for a "linear CI" expansion $\Psi_{\mathrm{gvb}}^{\text {linear }}$ in which the functionals $D_{i}\left(\left\{\sigma_{n m}\right\}\right)$ of Eq. (4) are replaced by coefficients $B_{i}$ which have free variational freedom,

$$
\Psi_{\mathrm{gvb}}^{\mathrm{linear}}=\sum_{i=1}^{2^{N_{\mathrm{gvb}}}} B_{i} \gamma_{i}
$$


Most importantly, as shown below, the GVB-PP wave function and generalizations of the GVB-PP wave function expressed in terms of the $\left\{\sigma_{n m}\right\}$. have energies which are nearly identical to that obtained by performing the larger linear CI. This computationally efficient method of treating the closed shell GVB-PP configurations will be shown below to be a very important tool for simplifying configuration expansions generated from a GVB-PP reference. Aside from these computational issues, the GVB wave function also has the important conceptual advantage that the local orbital representation allows for a compact, chemically interpretable wave function.

\section{RESTRICTED CONFIGURATION INTERACTION FROM A GVB-PP REFERENCE}

The RCI wave function is generated from the GVB-PP wave function by allowing for the open-shell spatial occupation $\phi_{i 1} \phi_{i 2}$ in each pair in addition to the two closed-shell GVB-PP occupations $\phi_{i 1}^{2}$ and $\phi_{i 2}^{2}$. In addition, for a given spin eigenstate, the various spin couplings among the openshell pairs are accounted for. The RCI wave function is thus a general multiconfigurational reference wave function which can both dissociate properly and account for general spin eigenstates. It has recently been shown ${ }^{5}$ that, as a reference wave function for multiconfigurational perturbation theory, the RCI wave function gives results comparable to results obtained ${ }^{6}$ with the much larger complete-active-space (CASSCF) reference wave function.

The RCI expansion we have formulated is constructed making intrapair excitations explicitly from the GVB-PP wave function of $\mathrm{Eq}_{\mathrm{q}}$ (3). This is in contrast to the $\mathrm{RCI}$ wave function previously constructed by others ${ }^{13,16}$ by making excitations from the linear CI expansion of the GVB-PP wave function [Eq. (6)]. Furthermore, following a suggestion of Ref. 18, we have found that an accurate approximation is to limit the number of excitations from the GVB-PP reference. By using the computational efficiencies of the GVB-PP reference discussed above, we presently show that a computationally efficient RCI wave function can be defined which allows for the correlation, of a large number of GVB pairs.

We first focus on the spatial aspects of the RCI wave function. The three possible spatial states of an "RCI pair" $i$ are defined as the GVB state $\xi_{i 0}$ and the two other pair states $\xi_{i 1}$, and $\xi_{i 2}$ which together form the orthogonal complement to $\xi_{i 0}$;

$$
\begin{aligned}
& \xi_{i 0}=\sigma_{i 1} \phi_{i 1}^{2}-\sigma_{i 2} \phi_{i 2}^{2} \ldots(\text { GVB-PP }), \\
& \xi_{i 1}=\phi_{i 1} \phi_{i 2} ; \quad \xi_{i 2}=\sigma_{i 2} \phi_{i 1}^{2}+\sigma_{i 1} \phi_{i 2}^{2} .
\end{aligned}
$$

In the GVB-PP wave function all pairs are in the $\xi_{i 0}$ state. The $\xi_{i 1}$ pair state is of the open-shell form and is orthogonal to $\xi_{i 0}$ via orthogonality of the natural orbitals. The $\xi_{i 2}$ state forms the other complement to $\xi_{i 0}$ with complementarity in this case defined with respect to the pair coefficient space $\sigma_{i}=\left(\sigma_{i 1}, \sigma_{i 2}\right)$. As discussed above [Eq. (5)], the optimization of the GVB-PP $\sigma_{i}$ gives two roots per pair, the GVB-PP root $\left(\sigma_{i 1},-\sigma_{i 2}\right)$ and the complementary root $\left(\sigma_{i 2}, \sigma_{i 1}\right)$. The $\xi_{i 2}$ pair state is formed from this complementary $\sigma_{i}$ solution.
The $\xi_{i 2}$ effectively adjust the $\sigma_{i}$ coefficients of $\xi_{i 0}$ due to the presence of the $\xi_{i 1}$ excitations in the RCI expansion.

In general, the RCI wave function constructed from a GVB-PP wave function of $N_{p}$ pairs, will allow only $N_{\text {rci }}$ of these pairs to be correlated beyond GVB-PP by occupation of all three of the possible pair states $\xi_{i j}$. The remaining $N_{\text {givbmf }}=\left(N_{p}-N_{\text {rci }}\right)$ pairs are forced to stay in the GVB-PP $\xi_{i 0}$ state. This restriction allows for correlation of a region of the system beyond the reference GVB-PP level, in the field of the remaining GVB-PP pairs. The utility of such a local specification of higher correlation is evident in many chemical problems such as correlation of dissociating bonds in the GVB-PP. field of the unperturbed bonds. In addition, we show below that the nature of the GVB-PP expansion allows for the $N_{\text {gvbmf }}$ GVB-PP pairs to be effectively represented as a single-particle mean field. Hence the nomenclature $N_{\text {gvbmf }}$ represents those pairs generating this GVB-PP mean field.

A spatial amplitude $\Phi_{r}^{\mathrm{RCI}}$ contributing to the $\Psi^{\mathrm{RCI}}$ wave function has the specified form,

$$
\Phi_{r}^{\mathrm{RCI}}=\{\operatorname{core}\}\left(\prod_{i=1}^{N_{\mathrm{gubmf}}} \xi_{i 0}\right)\left(\prod_{j=1}^{N_{\mathrm{rci}}} \xi_{j n}\right) \quad n=0,1,2
$$

where the specific spatial term $\Phi_{r}^{\mathrm{RCI}}$ is distinguished by the states $(n=0,1,2)$ of the $N_{\text {rci }}$ pairs and \{core\} denotes a closed-shell core. For $N_{\text {rci }}$ pairs it is possible to generate $3^{N_{\text {rei }}}$ such spatial terms $\Phi_{r}^{\mathrm{RCI}}$. However, we show below that it is possible to identify a subset of these spatial terms which are the most energetically important. This subset is specified by allowing a maximum number of excited pair states $\left(\xi_{i 1}\right.$ or $\left.\xi_{i 2}\right)$ to occur in any $\Phi_{r}^{\mathrm{RCI}}$.

Having specified the spatial terms $\Phi_{r}^{\mathrm{RCI}}$, configuration state functions $\psi_{n}^{\mathrm{RCI}}$ defining $\Psi^{\mathrm{RCI}}$ can be specified by assigning all possible spin eigenfunctions $\Theta_{k}$ to the spatial terms $\Phi_{r}^{\mathrm{RCI}}$,

$$
\psi_{n}^{\mathrm{RCI}}=\mathscr{A}\left[\Phi_{r}^{\mathrm{RCI}} \Theta_{k}\right] \text {, }
$$

The number of $\Theta_{k}$ assigned to a $\Phi_{r}^{\mathrm{RCI}}$ increases as a function of the number of $\xi_{i 1}$ open-shell RCI pairs in $\Phi_{r}^{\mathrm{RCI}}$ in the usual $^{19}$ fashion. The RCI wave function is now completely specified as an expansion in the configuration state functions $\psi_{n}^{\mathrm{RCI}}$ with variational weights $C_{n}$,

$$
\Psi^{\mathrm{RCI}}=\sum_{n} C_{n} \psi_{n}^{\mathrm{RCI}}
$$

Thus the RCI expansion is expressed in terms of configurations which are identified by the specific pairs which are excited beyond the GVB-PP level $\xi_{i 0}$, and the spin coupling among the open-shell $\xi_{i 1}$.

A very important and novel aspect of $\Psi^{\mathrm{RCl}}$ is the socalled contracted nature of the expansion. The RCI wave function is contracted in the sense that each variationally weighted entity $\psi^{\mathrm{RCI}}$ is in fact a sum of up to $2^{N_{\mathrm{gvb}}}$ individual uncontracted configuration state functions $\gamma_{i}$. The contraction of a given configuration is explicitly shown below, 
TABLE I. The number of contracted RCI configurations $N_{\text {con }}^{\text {RCI }}$ vs the number of uncontracted configurations $N_{\text {ucon }}$ contained in $\Psi^{\mathrm{RCI}}$ with $N_{\text {rci }}$ pairs.

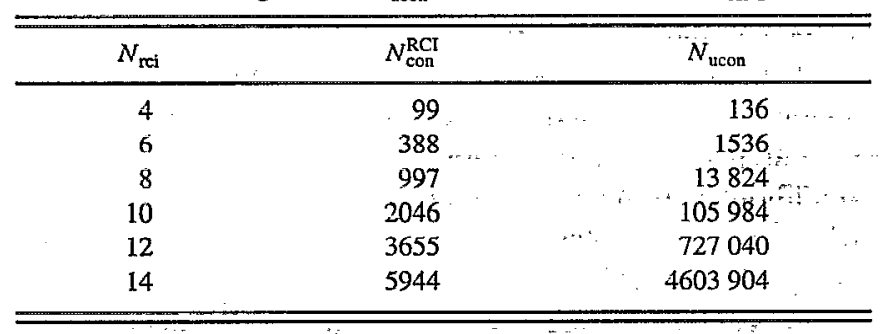

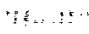

$$
\begin{aligned}
& \psi_{n}^{\mathrm{RCI}}=\ell\left[\Phi_{r}^{\mathrm{RCI}} \Theta_{k}\right]=\ell\left[\left(\prod_{j=1}^{N_{p} \cdot \xi_{j p}}\right)^{\top} \Theta_{k}\right] \\
& \left.=\sum_{m} A_{m}(\{\sigma\}) \cdot\left(\prod_{s} \phi_{n s}\right) \Theta_{k}\right] \\
& =\sum_{m} A_{m}(\{\sigma\}) \gamma_{m} .
\end{aligned}
$$

This explicitly shows the RCI configuration $\psi_{n}^{\mathrm{RCI}}$ as a weighted sum of order $2^{N_{p}}$ individual configurations labeled $\gamma_{m}$. This contraction of a given configuration is analogous to the orbital based contraction discussed above for GVB-PP. In conventional "linear" CI the $\gamma_{m}$ are the basis in which $\mathrm{CI}$ is performed. The contracted CI contains this full $\gamma_{m}$ basis but dóes not allow each $\gamma_{m}$ to independently vary. Instead, fixed linear combinations of the $\gamma_{m}$ vary as specified by the coefficients $A_{m}(\{\sigma\})$. The computational significance of this statement is that $\Psi^{\mathrm{RCI}}$ can be determined in a space with a dimension which is much smaller than the number of individual $\gamma_{m}$ contained in the expansion. To illustrate this large reduction in dimensionality afforded by the contraction procedure, we have tabulated in Table I the number of contracted RCI configurations vs the number of uncontracted configurations $\gamma_{m}$ in $\Psi^{\mathrm{RCI}}$ for $N_{p}=N_{\text {rci }}=4,14$ pairs. A singlet state was assumed and a limit of 'at most three nonGVB-PP pair states $\left(\xi_{i 1}\right.$ or $\left.\xi_{i 2}\right)$ in any $\psi_{n}^{\mathrm{RCI}}$ was imposed. The corresponding restriction of at most six open-shell orbitals (three open-shell pairs)' was imposed on the uncontracted expansion.

The dimension of the contracted space clearly grows with $N_{\text {rci }}$ at a much slower rate than that of the uncontracted space. This slow growth of the contracted space is a prerequisite to correlating much larger systems than previously possible. To realize this potential it is also necesssary to demonstrate an efficient scheme for calculating matrix elements among contracted RCI configurations, this is the topic of the next section. Second, we must address the accuracy of the contraction scheme relative to $\mathrm{CI}$ in the corresponding uncontracted space generated with the same restriction on the maximal number of open-shell orbitals as in the contracted RCI. Relative to this analogous uncontracted CI, the contraction scheme clearly involves the orbital based contraction approximation analogous to the GVB-PP case. Second, by placing an upper limit on the number of $\xi_{i 2}$ pair excitations, the contraction scheme is limiting the extent of reoptimiza- tion of the GVB pair coefficients $\left\{\sigma_{n m}\right\}$ caused by the openshell $\xi_{i 1}$ excitations. Test calculations presented below indicate that the contracted $\mathrm{CI}$ is indeed a very good approximation to the uncontracted $\mathrm{CI}$.

It is important to distinguish the contraction method outlined here with the contraction method traditionally used in multireference $\mathrm{CI}$ calculations. ${ }^{2}$ The latter multireference $\mathrm{CI}$ contraction is based on making excitations from an uncontracted MCSCF wave function with the resulting contracted $\mathrm{Cl}$ coefficients being functionals of the uncontracted MCSCF $\mathrm{CI}$ coefficients. In contrast, our contraction method is based on both making excitations from the GVB-PP reference and from the fact that the GVB-PP CI coefficients are contracted functions of the orbital expansion coefficients. $\left\{\sigma_{n m}\right\}$. This latter orbital based contraction of the closed-shell space proves to be very important for efficient generation of matrix elements among contracted RCI configurations and, as shown above, for reducing the length of the $\mathrm{CI}$ expansion.

\section{CONTRACTED RCI MATRIX ELEMENTS USING GVB-PP MEAN FIELDS}

Since each contracted RCI configuration contains a sum of up to $2^{N}$ uncontracted configurations, the calculation of Hamiltonian matrix elements among contracted RCI configurations could potentially require an expensive computation of many uncontracted matrix elements. Fortunately, the calculation of matrix elements among contracted $\mathrm{RCI}$ configurations is greatly simplified by the realization that the common nonexcited GVB-PP parts of two interacting configurations can be replaced by a single-particle Coulombexchange mean field operator. This simplification involves no further approximations beyond those made in the definition of the contracted RCI expansion. We presently discuss the details of contracted matrix element evaluation utilizing this GVB mean field procedure.

\section{A. Correlation in a GVB-PP mean field}

The simplest demonstration of this mean field approach considers the treatment of the $N_{\text {gvbmf }}$ unexcited GVB pairs in the RCI configurations of Eqs. (8) and (9). It is possible to write each contracted configuration $\psi_{n}^{\mathrm{RCI}}$ as an antisymmetrized product of the GVB-PP wave function $\Psi^{\mathrm{gvbmf}}$ which is common to all configurations $\psi_{n}^{\mathrm{RCI}}$, multiplied by the remaining part of the configuration, $\psi_{n}^{N_{\text {rci }}}$, describing the $N_{\text {rci }}$ pairs correlated beyond GVB-PP,

$$
\begin{aligned}
& \left.\psi_{n}^{\mathrm{RCI}}=\psi^{\mathrm{gvbmf}} \psi_{n}^{N_{\mathrm{rci}}}\right] ; \cdots \\
& \Psi^{\mathrm{gvbmf}}=\left[\left(\prod_{i=1}^{N} \prod_{i 0}^{\mathrm{gvbmf}} \xi_{i}\right)(\alpha \beta \ldots \alpha \beta)\right] \text {, } \\
& \psi_{n}^{N_{\mathrm{rci}}}=A\left[\Phi_{r}^{N_{\mathrm{rci}} \Theta_{k}}\right]
\end{aligned}
$$

In these equations $\Psi^{\mathrm{gvbmf}}$ and $\psi_{n}^{N_{\text {rel }}}$ are defined in the space of the $2 N_{\text {gubmf }}$ and $2 N_{\text {rci }}$ coordinates, respectively. The antisymètrizer of $\psi_{n}^{\mathrm{RCI}}$ permutes between these sets of coordinates. Since $\Psi^{\mathrm{gubmf}}$ is common to all configurations, $\Psi^{\mathrm{RCl}}$ can be factorized into $\Psi^{\text {gvbmf }}$ and the expansion in the space of $N_{\text {rci }}$ pairs, $\Psi_{\text {rci, }}^{N_{\text {ri }}}$ 


$$
\Psi^{\mathrm{RCI}}=A\left[\Psi^{\mathrm{gvbmf}} \Psi^{N_{\mathrm{rci}}}\right] ; \quad \Psi^{N_{\mathrm{rci}}}=\sum_{n} C_{n} \psi_{n}^{N_{\mathrm{rci}}}
$$

This factorization is possible since $\Psi^{\text {gvbmf }}$ and $\Psi^{N_{\text {rei }}}$ do not share any common spatial orbitals. Furthermore, the CI coefficients $C_{n}$ of Eq. (13) are the same as those of Eq. (4) since $\Psi^{\text {gvbmf }}$ is normalized independently of $\Psi^{N_{\text {ret }}}$ by the pairwise normalization of a GVB-PP wave function [Eq. (2)].

Using this factorization and the fact that $\Psi^{\text {gvbmf }}$ is a weighted sum of closed shell determinants [Eq. (4)]; a straightforward generalization of the hypothetical limit in which $\Psi^{g v b m f}$ is a single determinant gives the following simplified energy expression for $\Psi^{\mathrm{RCI}}$,

$$
\begin{aligned}
\left\langle\Psi^{\mathrm{RCI}}|\mathscr{\mathscr { C }}| \Psi^{\mathrm{RCI}}\right\rangle= & \left\langle\Psi^{\mathrm{gvbmf}}|\mathscr{H}| \psi^{\mathrm{gvbmf}}\right\rangle \\
& +\left\langle\Psi^{N_{\mathrm{ric}} \mid \mathscr{F}^{*}} \mid \Psi^{N_{\text {ric }}}\right\rangle .
\end{aligned}
$$

The first matrix element is the GVB-PP energy of $\Psi^{\text {gvbinf }}$ and the second matrix element is the energy of $\Psi^{N_{\text {rci }}}$ evaluated with a new effective Hamiltonian $\mathscr{H}^{*}$. This effective Hamiltonian contains the full Hamiltonian $\mathscr{Z}$ and the gencralized Fock operator ${ }^{3,20} F^{\text {gvbmf }}$ of $\Psi^{\text {gvbmf }}$,

$$
\mathscr{H} *=\mathscr{H}+F^{\mathrm{gvbmf}} ; \quad F^{\mathrm{gvbmf}}=\sum_{m} \sigma_{m}^{2}\left(2 J_{m m}-K_{m m}\right) .
$$

The $F^{\text {gvbmf }}$ single-particle operator is simply a weighted sum of closed-shell Fock operators for orbitals $m$ contained in $\Psi^{\text {gvbmf }}$. Computationally, $F^{\text {gvbmf }}$ can be treated as a single conventional closed-shell Fock operator in the atomic orbital space using the closed-shell single-particle atomic-orbital density matrix of $\Psi^{\mathrm{gvbmf}}$.

This derivation conceptually and computationally extends the traditional approach of correlating a spatial region in a surrounding Hartree-Fock mean field to the use of a correlated GVB-PP mean field. This GVB mean field significantly reduces the computational effort of the RCI calculation by replacing the $2^{N_{\text {gvbmf }}}$ set of configurations in $\Psi^{\text {gvbmf }}$ by one single-particle operator. The two-electron part of the Hamiltonian is therefore effectively reduced to the space of the $2 N_{\text {rci }}$ coordinates. Calculations presented below show that $F^{\mathrm{gvbm}}$ accurately represents the effects of an uncontracted expansion in $2^{N_{\text {gvbmf }}}$ configurations multiplying the expansion $\Psi^{N_{\text {rci. }}}$

\section{B. Matrix elements in the $\mathrm{RCl}$ space}

Given that we have effectively integrated the static or unexcited $\Psi^{\text {gvbmf }}$ space out of the correlation problem, the remaining problem is to efficiently formulate the matrix elements in the excited RCI space between the $\psi_{n}^{N_{\text {rci }}}$ contracted configurations. We presently describe how the efficiencies of the GVB mean field approach can be extended to the construction of contracted matrix elements in the RCI space.

The nonzero matrix elements between configurations $\psi_{n}^{N_{\text {rei }}}$ and $\psi_{m}^{N_{\text {rei }}}$ of Eq. (12) can be classified in the usual fashion ${ }^{21}$ based upon the difference between the contracted spatial configurations $\Phi_{n}^{N_{\text {rci }}}$ and $\Phi_{m}^{N_{\text {rci }}}$. The four classes of matrix elements are: (a) The diagonal case where $\Phi_{n}=\Phi_{m}$, (b) the class for which $\Phi_{n}$ and $\Phi_{m}$ differ by an intrapair single excitation, e.g., $\Phi_{n}$ is in the $\xi_{j 0}$ state and $\Phi_{m}$ in the $\xi_{j 1}$ state, (c) the case when $\Phi_{n}$ and $\Phi_{m}$ differ by an intrapair double excitation, and (d) the case when $\Phi_{n}$ and $\Phi_{m}$ differ by intrapair single excitations at two pair sites. The RCI Hamiltonian is made relatively sparse by the fact that the RCI pairs do not share common natural orbitals and by the intrapair nature of the RCI excitations.

Since the $\psi_{n}^{N_{\text {rei }}}$ are composed of up to $2^{N_{\text {rci }}}$ uncontracted configurations, it is too expensive to simply expand each configuration into uncontracted configurations and then sum matrix elements among uncontracted configurations. The key to avoiding this $2^{N_{\text {rci }}}$ growth in computational labor is once again the use of the GVB mean field approach, and the imposition of a limit on the maximum number of excitations from the GVB $\xi_{i 0}$ state in the space of RCI pairs. For the first three classes of matrix elements between configurations $\psi_{n}^{N_{\text {rci }}}$ and $\psi_{m}^{N_{\text {rci }}}$ listed above the following approach is adopted. The first step is to identify the set of $N_{n m}^{0}$ pairs in the interacting spatial configurations $\Phi_{n}^{N_{\text {rci }}}$ and $\Phi_{m}^{N_{\text {rci }}}$ which are in the GVB-PP state $\xi_{i 0}$ in both $\Phi_{n}^{N_{\text {rci }}}$ and $\Phi_{m}^{N_{\text {rci }}}$. The remaining $N_{n m}^{\mathrm{ex}}=\left(N_{\text {rci }}-N_{n m}^{0}\right)$ excited pairs must satisfy the condition that the pairs are in the excited $\xi_{i 1}$ or $\xi_{i 2}$ state in $\Phi_{n}^{N_{\text {ri }}}$ and/or $\Phi_{m}^{N_{\text {rci }}}$. As in the analysis of the static GVB mean field above we now rewrite $\psi_{n, m}^{N_{\text {ri }}}$ with their common GVB-PP space of $N_{n m}^{0}$ pairs factored out into a GVB-PP wave function $\psi_{n m}^{\mathrm{gvbmf}}$ and into the remaining excited terms $\psi_{n}^{\mathrm{ex}}$ and $\psi_{m}^{\mathrm{ex}}$ defined in the defined in the space of $N_{n m}^{\mathrm{ex}}$ pairs,

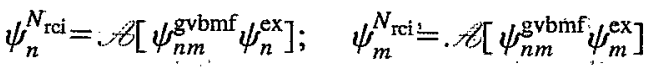

$$
\begin{aligned}
& \psi_{n m}^{\mathrm{gvbmf}}=\not\left[\left(\prod_{i=1}^{N_{n m}^{0}} \xi_{i 0}\right)(\alpha \beta \ldots \alpha \beta)\right]
\end{aligned}
$$

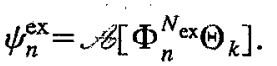

The $\Phi_{n}^{N_{\mathrm{ex}}}$ and $\Theta_{k}$ spatial and spin terms defining the reduced configurations $\psi_{n}^{\mathrm{ex}}$ are defined in the space of the $N_{n m}^{\mathrm{ex}}$ contracted pairs. In a manner analogous to the derivation of $F^{\text {gvbmf }}$ it is readily shown that the following expression applies to the matrix element between $\psi_{n}^{N_{\text {rci }}}$ and $\psi_{m}^{N_{\text {rci }}}$,

$$
\begin{aligned}
\left\langle\psi_{n m}^{\mathrm{gvmf}} \psi_{n}^{\mathrm{ex}}\left|\mathscr{E}^{*}\right| \psi_{n m}^{\mathrm{gvbmf}} \psi_{m}^{\mathrm{ex}}\right\rangle= & \delta_{n m}\left\langle\psi_{n m}^{\mathrm{gvbmf}}\left|\mathscr{F}^{*}\right| \psi_{n m}^{\mathrm{gvbmf}}\right\rangle \\
& +\left\langle\psi_{n}^{\mathrm{ex}}\left|\mathscr{F}_{n m}^{*}\right| \psi_{m}^{\mathrm{ex}}\right\rangle
\end{aligned}
$$

where the effective Hamiltonian $\mathscr{R}_{n m}^{*}$ is given by,

$$
\mathscr{L}_{n m}^{*}=\mathscr{P}^{*}+F_{n m}^{\mathrm{gvbmf}} ; \quad F_{n m}^{\mathrm{gvbmf}}=\sum_{q} \sigma_{q}^{2}\left(2 J_{q q}-K_{q q}\right) .
$$

The orbitals $q$ are those defining $\psi_{n m}^{\text {gvhmf }}$ and $\mathscr{F}$ includes the static GVB Fock operator of Eq. (15). The single-particle Fock operator $F_{n m}^{\text {gubmf }}$ effectively represents the GVB-PP mean field common to $\psi_{n}^{\mathrm{RCI}}$ and $\psi_{m}^{\mathrm{RCI}}$.

The evaluation of the contracted RCI matrix element has therefore been reduced to the space of $N_{n m}^{\mathrm{ex}}$ contracted RCI pairs using $\mathscr{H}_{n m}^{*}$. The matrix element in the $N_{n m}^{\text {ex }}$ space is of 
one of the four classes noted above with, by definition, the difference between spatial terms $\Phi_{n}^{N_{\text {rci }}}$ and $\Phi_{m}^{N_{\text {rci }}}$ occurring at one or two of the $N_{n m}^{\mathrm{ex}}$ pair sites. The matrix element in the $N_{n m}^{\text {ex }}$ space is,

$$
\left\langle\psi_{n}^{\mathrm{ex}}\left|\mathscr{K}_{n m}^{*}\right| \psi_{m}^{\mathrm{ex}}\right\rangle=\left\langle\xi_{1}^{n} \ldots \xi_{N_{n m}^{\mathrm{ex}}}^{n} \Theta_{r}\left|\mathscr{C}_{n m}^{*}\right| \sim \mathcal{Z}^{2}\left[\xi_{1}^{m} \ldots \xi_{N_{n m}^{\mathrm{ex}}}^{m} \Theta_{s}\right]\right\rangle
$$

Since the pair functions $\xi_{i j}$ are orthonormal, this contracted matrix element can be evaluated in the same manner as an uncontracted matrix element. However, interactions involving $\xi_{i 0}$ and $\xi_{i 2}$ require additional summations over the two closed-shell orbitals of these pair functions. The net matrix element is a sum of uncontracted matrix elements weighted by products of $\left\{\sigma_{m m}\right\}$ coefficients. Note however that the class (c) type of uncontracted matrix element involves a difference of diagonal matrix elements and hence requires, unlike the uncontracted case, the evaluation of $F_{\mathrm{mm}}^{\mathrm{gvbm}}$ while the contracted matrix element of type (d) involves, as in the uncontracted case, no one-particle component.

In summary, by using the GVB mean field approach the two-electron part of contracted matrix elements has been effectively reduced to the space of $N_{n m}^{\mathrm{ex}}$ pairs. Furthermore, since we have a limit of order three on the number of nonGVB pairs in a given configuration, the maximum size of the $N_{n m}^{\mathrm{ex}}$ space is of order six pairs and on average $N_{n m}^{\mathrm{ex}}$ is two to three pairs. Thus the cost of evaluating the sums of uncontracted two-electron matrix elements in Eq. (20) is significantly reduced by this reduction in the size of the $N_{n m}^{\mathrm{ex}}$ space.

\section{PSEUDOSPECTRAL INTEGRAL EVALUATION}

The RCI matrix elements discussed above can be expressed in terms of the Coulomb and exchange operators $J_{i j}$ and $K_{i j}$ where $i, j$ refer to the three possible orbital products of each RCI pair, $\phi_{i 1} \phi_{i 1}, \phi_{i 2} \phi_{i 2}, \phi_{i 1} \phi_{i 2}$. The matrix elements of these operators between atomic basis functions $\chi_{\mu}$, $\chi_{\nu}$, have the usual form,

$$
\begin{aligned}
& \left\langle\mu\left|J_{i j}\right| \nu\right\rangle=\left(\mu(1) \nu(1) \mid \phi_{i}(2) \phi_{j}(2)\right) ; \\
& \left\langle\mu\left|K_{i j}\right| \nu\right\rangle=\left(\mu(1) \phi_{i}(1) \mid \nu(2) \phi_{j}(2)\right) .
\end{aligned}
$$

The pseudospectral representation of a Coulomb matrix element is represented by,

$$
\left\langle\mu\left|J_{i j}\right| \nu\right\rangle=\sum_{g} Q_{\mu}(g) J_{i j}(g) R_{\nu}(g)
$$

The sum is over grid points $g . J_{i j}(g)$ is the physical space representation of $J_{i j}$ given by a sum of three-center, oneelectron integrais weighted by the orbital expansion coefficients $c_{k}^{i}$

$$
J_{i j}(g)=\sum_{k l} c_{k}^{i} c_{l}^{j} \int \frac{\chi_{k}(1) \chi_{l}(1)}{r_{1 g}} d \mathbf{r}_{1} .
$$

The term $R_{\nu}(g)$ is simply the basis function at grid point $g$, $\chi_{\nu}(g)$. Finally, the term $Q_{\mu}(g)$ results from the least squares fit of $J_{i j}(g) \chi_{\nu}(g)$ to the atomic orbital basis augmented by a dealiasing basis. Similar expressions apply to the exchange operator. Further details regarding the the construction of the pseudospectral quantities can be found in Refs. 7-12. To attain higher accuracy, a limited number of two-electron integrals contributing to $J_{i j}^{\mu \nu}$ and $K_{i j}^{\mu \nu}$ are evaluated analytically as discussed in Ref. 11 .

Using $N_{\text {bas }}$ basis functions $\{\chi\}$ in which to expand the orbitals $\phi_{i}$, the pseudospectral evaluation of each Coulomb and exchange operator over this basis scales as $N_{\text {bas }}^{3}$ as compared to the conventional analytic scaling of $N_{\text {bas }}^{4}$. Second, the formation of the $\mathrm{RCl}$ matrix elements requires that the Coulomb and exchange operators be transformed from the atomic basis $J_{i j}^{\mu \nu}$ to the natural orbital basis $J_{i j}^{k l}$ by a simple two-index transform,

$$
\left\langle\phi_{k}\left|J_{i j}\right| \phi_{l}\right\rangle=\sum_{\mu \nu} c_{\mu}^{k} c_{\nu}^{l} J_{i j}^{\mu \nu}
$$

This two-index transformation thus avoids the expensive conventional four-index transform from the integrals over basis functions to integrals over orbitals which is a major bottleneck (an $N_{\text {bas }}^{5}$ procedure) in conventional MCSCF and $\mathrm{CI}$ approaches.

\section{TEST RESULTS}

In the following sections we are primarily interested in demonstrating the accuracy of the pseudospectral contracted $\mathrm{RCI}$ expansion relative to the corresponding conventional uncontracted expansion. In addition we present some representative examples of the improvements in spectroscopic parameters obtained by RCI relative to GVB-PP. Finally we discuss the computational speed of the $\mathrm{RCI}$ program for larger problems.

\section{A. Details of calculations}

The self-consistent pseudospectral GVB-PP calculations were performed with the PS-GVB program of Ref. 12. The contracted pseudospectral RCI program was written in a direct-CI manner to iteratively ${ }^{22}$ diagonalize the full Hamiltonian over contracted RCI configurations. The main task of the RCI program is to generate matrix-vector products of contracted RCI matrix elements. The pseudospectral RCI integrals of Eq. (22) were produced by a modification of the PS-GVB program. All contracted RCI results used a maximum of three pair excitations from the GVB-PP reference. The uncontracted pseudospectral RCI calculations were done with the uncontracted version of the contracted code while the uncontracted RCI calculations using all analytic integrals were done with the programs of Ref. 23.

The RCI calculations were performed with the so-called "ultra-fine" grid of the PS-GVB program. This grid has approximatcly 425 points per atom. Analytic atom and diatomic corrections were used as explained in Ref. 11. We have found that it is possible to limit these corrections of the matrix elements involving a GVB pair to basis functions on the two atoms defining the pair. This intrapair correction approach is currently implemented for the GVB Coulomb and exchange matrix elements and the $\mathrm{RCI}$ exchange matrix elements in the CPU timings below. 
TABLE II. Convergence of RCI energy with maximum open-shell occupation.

\begin{tabular}{ccccccc}
\hline \hline & \multicolumn{5}{c}{ Maximum open-shell pairs } \\
\cline { 2 - 6 } Molecule & 2 & \multicolumn{3}{c}{3} & \multicolumn{2}{c}{4} \\
\hline$N_{2}$ & 0.068045 & 0.068088 & 0.068604 & 0.068604 & $\ldots$ \\
Ethane & 0.028991 & 0.029018 & 0.029191 & 0.029191 & 0.029191 \\
$\mathrm{CH}_{3} \mathrm{C} N$ & 0.067121 & 0.079295 & 0.079575 & 0.079586 & 0.079602 \\
Glyoxal & 0.051784 & 0.051814 & 0.052464 & 0.052464 & 0.052466 \\
Ethanol & 0.026867 & 0.026892 & 0.027034 & 0.027034 & 0.027034 \\
\hline \hline
\end{tabular}

The tests of the contracted method used $631 \mathrm{G}^{* *}$ basis sets. The diatomic calculations in Sec. VIE used correlation consistent ${ }^{24} 4 s 3 p 2 d$ basis sets on $\mathrm{C}, \mathrm{N}, \mathrm{O}$ and a $3 s 2 p$ set on H.

\section{B. Test of open-shell restriction}

As discussed in Sec. IV B, the computational cost of the $\mathrm{RCI}$ expansion can be significantly reduced by restricting the maximum number of contracted pair excitations to a maximum level, nopmx, within any configuration. The corresponding statement in the uncontracted $\mathrm{CI}$ is that we limit the $\mathrm{CI}$ to at most $2^{*}$ nopmx open-shell orbitals. This restriction in the contracted expansion not only places the same limit on the maximum open-shell occupation, but also limits the reoptimization of the pair coefficients $\{\sigma\}$ since the maximum number of $\xi_{i 2}$ pairs is also nopmx. As discussed above this restriction is one of the main approximations of the contraction method. In this section we present uncontracted RCI results for some small systems to support a limit on the openshell occupations. The results of the next section comparing contracted and uncontracted results will test the remaining approximations of a limit on the $\xi_{i 2}$ type excitations and the accuracy of the orbital based contraction.

The convergence of the uncontracted RCI energy with maximum number of open-shell pairs is shown in Table II for some small molecules. All valence pairs were correlated except for the ethanol $\mathrm{OH}$ bond due to limitations of the uncontracted CI program. In some cases most or all of the extra configurations generated by a larger open shell number do not have the correct symmetry and hence the RCI energy does not change appreciably. For this reason the $\mathrm{CH}_{3} \mathrm{CN}$ molecule with the lowest symmetry is perhaps the best example. The use of three open shell pairs recovers $98 \%$ or more of the six open-shell RCI correlation energy relative to GVB-PP while four open-shell pairs is nearly indistinguishable from six. From these results and others ${ }^{18}$ it is plausible to assume that for organic molecules near their equilibrium geometries that at most three or four open-shell pairs in any given configuration yield sufficient accuracy.

\section{Accuracy of contraction}

The accuracy of the pseudospectral contracted RCI expansion was calculated relative to the corresponding uncontracted RCI expansion for the molecules in Table III using a self-consistent GVB-PP reference obtained pseudospectrally.
TABLE III. Accuracy of contracted RCI relative to uncontracted RCI. $\Delta E$ is the energy difference between the contracted and uncontracted wave functions. $N_{\text {pair }}$ is the number of RCI pairs correlated:

\begin{tabular}{ccc}
\hline Molecule & $N_{\text {pair }}$ & $\Delta E(\mathrm{kcal} / \mathrm{mol})$ \\
\hline $\mathrm{H}_{2} \mathrm{O}$ & 4 & 0.014 \\
$\mathrm{C}_{2} \mathrm{H}_{2}$ & 5 & 0.018 \\
$\mathrm{C}_{2} \mathrm{H}_{4}$ & 6 & 0.022 \\
$\mathrm{C}_{2} \mathrm{H}_{6}$ & 7 & 0.047 \\
Glyoxal & 7 & 0.049 \\
Acetic acid & 8 & 0.034 \\
Ethanol & 8 & 0.046 \\
Acetone & 10 & 0.005 \\
\hline
\end{tabular}

The contracted RCI was performed with a restriction of at most three pair excitations relative to the GVB-PP reference. The uncontracted RCI used the pseudospectral RCI integrals and a maximum open-shell occupation of three pairs. As discussed above, the contracted expansion contains all of the configurations of the uncontracted expansion. This comparison tests the approximations of the contraction procedure, namely the restricted reoptimization of the GVB-PP $\{\sigma\}$ parameters in the presence of the open-shell pair excitations and the accuracy of the orbtal based contraction.

For up to ten pairs the difference between the contracted and uncontracted RCI energies is seen to be negligible. Tests on systems larger than ten pairs were not attempted due to the unmanagable lengths of the uncontracted RCI expansion for larger pair number. However, there is no apparent growth in the contracted-uncontracted energy difference with pair number for these examples. These results indicate that the approximations made in the contracted method are indeed very reliable.

\section{Accuracy of pseudospectral integration}

Hartree-Fock and GVB-PP wave functions evaluated pseudospectrally with limited one- and two-center analytic integral corrections have been previously shown ${ }^{11}$ to have errors of order $0.01-0.04 \mathrm{kcal} / \mathrm{mol}$ relative to the corresponding all analytic integral calculations. We presently show that a similar accuracy applies to the pseudospectral RCI integral evaluation. Table IV presents a comparison between GVB-PP and uncontracted RCI calculations with all analytic integral evaluation vs pseudospectral integral evaluation. The error incurred by the pseudospectral evaluation of the RCI integrals is clearly very small, of order $0.05 \mathrm{kcal} /$ mol. It should be noted that this accuracy of the pseudospec-

TABLE IV. Accuracy of pseudospectral RCI integration. Uncontracted analytic RCI energies $E(A N-R C I)$ vs uncontracted pseudospectral energies $E$ (PS-RCI) (a.u.).

\begin{tabular}{cccc}
\hline Molecule & $E($ AN-RCI $)$ & $E($ PS-RCI $)$ & $\Delta(\mathrm{kcal} / \mathrm{mol})$ \\
\hline $\mathrm{C}_{2} \mathrm{H}_{4}$ & -78.167882 & -78.167917 & 0.022 \\
$\mathrm{C}_{2} \mathrm{H}_{6}$ & -79.372059 & -79.372058 & 0.001 \\
Ethanol & -154.256560 & -154.256470 & 0.056 \\
Glyoxal & -226.785405 & -226.785358 & $0.030^{*}$ \\
Acetic acid & -228.002985 & -228.002988 & 0.002 \\
\hline \hline
\end{tabular}


TABLE V. GVB-PP and RCI bond lengths $(\AA)$ and harnonic frequencies $\left(\mathrm{cm}^{-1}\right)$.

\begin{tabular}{ccccccc}
\hline Molecule & GVB $R_{e}$ & RCI $R_{e}$ & Expt. $R_{e}$ & GVB $\omega_{e}$ & RCI $\omega_{e}$ & Expt. $\omega_{e}$ \\
\hline $\mathrm{CO}$ & 1.119 & 1.129 & 1.128 & 2331 & 2179 & 2170 \\
$\mathrm{~F}_{2}$ & 1.485 & 1.422 & 1.413 & 675 & 856 & 917 \\
$\mathrm{~N}_{2}$ & 1.092 & 1.098 & 1.098 & 2413 & 2388 & 2359 \\
\hline
\end{tabular}

tral method is only obtainable by using the limited set of analytic corrections in the pseudospectral procedure. Recent pseudospectral calculations ${ }^{25}$ of CI and MP2 energies without such corrections gave non-negligible differences of order $0.7 \mathrm{kcal} / \mathrm{mol}$ from the analytic results. We have found that the corrections of integrals involving orbitals of a GVB pair can be limited to basis functions on the two atoms defining the GVB pair.

\section{E. Diatomic tests, $\mathrm{RCl}$ properties}

In addition to the above construction of an efficient RCI methodology for large molecules, an equally important task is to understand the quality of this wave function for predicting molecular properties. As a preliminary step in this direction we present some representative examples of the improvements in diatomic potential parameters obtained by RCI relative to GVB-PP. Lacking RCI gradient capabilities at this time we are limited to these simple examples. Table $\mathrm{V}$ presents the GVB-PP and RCI potential parameters for multiply bonded molecules $\mathrm{CO}, \mathrm{N}_{2}$, and $\mathrm{F}_{2}$. The $\mathrm{RCI}$ wave function clearly corrects the substantial errors made by GVB-PP.

A more thorough study of the predictive properties of the $\mathrm{RCI}$ wave function will be the focus of future work. Some other representative uncontracted $\mathrm{RCI}$ calculations by Goddard et al. can be found in Refs. 13 and 16. In general, we expect that it will be necessary to go beyond the RCI approximation to reliably obtain quantitative accuracy for molecular observables including dissociation energies and van der Waals interactions which are know to require high levels of correlation. In particular, we intend to use the RCI wave function as a general reference to be corrected by generalized Møller-Plesset perturbation theory. This multiconfigurational perturbation theory from an RCI or CASSCF wave function has recently been shown $\mathrm{w}^{3-6}$ to be a very accurate and computationally efficient approach. In addition it has been shown ${ }^{5}$ that generalized Møller-Plesset observables calculated with an RCI reference are comparable to those obtained with the much larger CASSCF reference. ${ }^{6}$

\section{F. Timings}

To provide a scale for the computational requirements of the GVB-RCI method we have performed calculations on the molecules in Table VI involving up to 35 GVB-RCI pairs and 250 basis functions. The GVB computations and RCI integrals were performed on an IBM-370 work station while the RCI energy was calculated on an IBM-580 which is roughly 1.5 times as fast as the IBM-370 for this code. All GVB integrals and RCI exchange integrals were done with a limited set of intrapair analytic corrections. To converge the
TABLE VI. CPU times (minutes) for self-consistent GVB (T-GVB), RCT integral evaluation (T-RCINT), and RCI energy evaluation (T-ERCI) for molecules with $N_{\text {bas }}$ basis functions and $N_{\text {pair }}$ electron pairs. $N_{\text {cnr }}$ is the number of contracted RCI configurations.

\begin{tabular}{lcccccc}
\hline \hline Molecule & $N_{\text {bas }}$ & $N_{\text {pair }}$ & T-GVB & T-RCINT & T-ERCI & $N_{\text {có }}$ \\
\hline Dimet. glyoxal & 120 & 17 & 41 & 20 & 3 & 10915 \\
Alanine & 160 & 23 & 140 & 50 & 10 & 14428 \\
Leucine & 200 & 26 & 310 & 115 & 13 & 40678 \\
Arginine & 250 & 35 & 650 & 224 & 60 & 101221 \\
\hline \hline
\end{tabular}

GVB energy to $5 \times 10^{-5}$ hartrees requires from 10 to 13 iterations for these examples. The GVB time is dominated by the integral evaluation. Normalizing the GVB CPU times linearly with respect to the number of GVB pairs and the number of iterations to convergence. the GVB CPU time displays an $N_{\text {bas }}^{2.5}$ scaling. The RCI integration time has a nearly equivalent scaling. The RCI energy evaluation time is well within the time required to evaluate the RCI integrals. The RCI energy calculation time scales with the number of RCI pairs $N_{\text {rci }}$ approximately as $N_{\text {rci }}^{3.8}$, however, this is a preliminary result as the $\mathrm{RCI}$ energy code requires further optimization.

Although the CPU times can certainly be reduced by further optimization, it is apparent from these examples that the pseudospectral GVB-RCI method can be applied to large molecules within manageable CPU times. It is important to stress that for many applications one would not need to correlate all pairs of a molecule but rather a small subset of pairs involved in changes from an initial to final state. The local nature of the GVB pairs greatly simplifies the assignment of the chemically important pairs.

\section{CONCLUSION}

In conclusion, pseudospectral integral evaluation combined with a novel GVB based contracted CI method has been shown to be a feasible approach for performing large correlated calculations within manageable CPU times. Much work remains to be done in both the optimization of the current approaches and in their extension to more accurate methods correlation. We intend to extend the contracted CI procedure to the generalized Møller-Plesset theory using the contracted RCI. wave function as a reference. Such a generalized Moller-Plesset theory is optimally implemented within the pseudospectral formalism in conjunction with the local virtual orbital methodology of Pulay. ${ }^{26}$ One of the most important conclusions to be drawn from this and related works is that an essential computational and conceptual simplifying element of all of these methods is the use of a local orbital basis.

\section{ACKNOWLEDGMENTS}

This research was supported by grants from NSF and NIH. M. N. R. thanks NIH for SBIR Grant No. 2 R44 GM46160-02.

\footnotetext{
'Reviews of the MCSCF method are presented in Adv. Chem. Phys. 69, 399 (1987).

${ }^{2}$ H. J. Werner and P. J. Knowles, J. Chem. Phys. 89, 5803 (1988).
} 
${ }^{3}$ K. Wolinski, H. L. Sellers, and P. Pulay, Chem. Phys. Lett. 140, 225 (1987).

${ }^{4}$ R. B. Murphy and R. P. Messmer, Chem. Phys. Lett. 183, 443 (1991).

${ }^{5}$ R. B. Murphy and R. P. Messmer, J. Chem. Phys. 97, 4170, 4974 (1992).

${ }^{6}$ K. Andersson, P. A. Malmqvist, and B. O. Roos, J. Chem. Phys. 96, 1218 (1992).

${ }^{7}$ R. A. Friesner, Chem. Phys. Lett. 116, 39 (1985).

${ }^{8}$ R. A. Friesner, J. Chem. Phys. 85, 1462 (1986).

${ }^{9}$ R. A. Frièsner, J. Chem. Phys. 86, 3522 (1987).

${ }^{10}$ R. A. Friesner, J. Phys. Chem. 92, 3091 (1988).

${ }^{11}$ J. M. Langlois, R. P. Muller, T. R. Coley, W. A. Goddard III, M. N. Ringnalda; , Y. W. Won, and R. A. Friesner, J. Chem. Phys. 92, 7488 (1990).

${ }^{12}$ M. N. Ringnalda, J. Langlois, B. H. Greeley, T. V. Russo, R. P. Muller, R. B. Murphy, B. Marten, R. E. Donnelly, Jr., W. T. Pollard, G. H. Miller, W. A. Goddard III, and R. A. Friesner (Schrödinger Inc., Pasadena CA).

${ }^{13}$ L. B. Harding and W. A. Goddard III, J. Am. Chem. Soc. 97, 6293 (1975).

${ }^{14}$ L. M. Cheung, K. R. Sundberg, and K. Ruedenberg, Int. J. Quantum Chem. 16, 1103 (1979).
${ }^{15}$ B. O. Roos, P. R. Taylor, and P. E. M. Siegbahn, Chem. Phys. 48, 157 (1980).

${ }^{16}$ E. A. Carter and W. A. Goddard IIT, J. Chem. Phys. 86, 862 (1987).

${ }^{17} \mathrm{~F}$. W. Bobrowicz and W. A. Goddard, in Methods of Electronic Structure Theory, edited by H. F. Schaefer III (Plenum, New York, 1977), p. 79.

${ }^{18}$ W. A. Goddard III, unpublished results.

${ }^{19} \mathrm{R}$. Pauncz, Spin Eigenfunctions-Construction and Use (Plenum, New York, 1979).

${ }^{20}$ R. B. Murphy and R. P. Messmer, J. Chem. Phys. 98, 10102 (1993).

${ }^{21}$ W. I. Salmon and K. Ruedenberg, J. Chem. Phys. 57, 2776 (1972).

${ }^{22}$ E. R. Davidson, J. Comp. Phys. 17, 87 (1975).

${ }^{23}$ The GVB-2 5 program provided by W. A. Goddard III, California Institute of Technology, was used for the uncontracted all analytic integral RCI calculations.

${ }^{24}$ T. H. Dunning, J. Chem. Phys. 90, 1007 (1989).

${ }^{25}$ T. J. Martinez and E. A. Carter, J. Chem. Phys. 98, 7081 (1993).

${ }^{26}$ P. Pulay, Chem. Phys. Lett. 100, 151 (1983). 